\title{
18. EMV-Fachtagung in Österreich
}

\author{
K. Lamedschwandner OVE IEEE
}

Online publiziert am 7. April 2020

C Springer-Verlag GmbH Austria, ein Teil von Springer Nature 2020

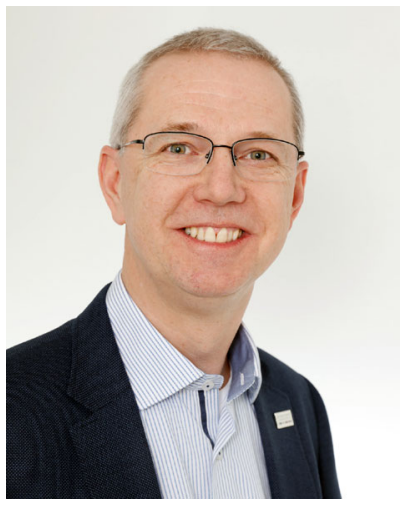

Dipl.-Ing. Dr. techn. Kurt Lamedschwandner
Liebe Leserinnen, liebe Leser!

Zeitgleich mit dem Erscheinen dieses e\&iHefts hätte die 18. EMVFachtagung in Seibersdorf stattfinden sollen. Aber leider ist jetzt aufgrund der Corona-Krise alles anders und wir mussten einen Ersatztermin finden.

Wir hoffen nun sehr, dass die österreichische EMVFachtagung zum neuen Termin am 30. September und 1. Oktober 2020 stattfinden kann, turnusgemäß organisiert von der Seibersdorf Labor GmbH in fachlicher Zusammenarbeit mit dem OVE Österreichischen Verband für Elektrotechnik, dem Institut für Elektronik der TU Graz und dem Austria Chapter der IEEE EMC Society.

Es ist mir eine große Ehre, dass ich parallel zur Vorbereitung der EMV-Fachtagung auch eine e\&i-Ausgabe zum Thema EMV koordinieren durfte, und es freut mich sehr, dass fünf Originalbeiträge und fünf Beiträge in der Rubrik Praxis und Wissen zur Veröffentlichung angenommen werden konnten. Dafür möchte ich mich bei der Autorin und allen Autoren sowie jenen Fachkolleg/innen, welche die Aufgabe übernommen haben, Beiträge zu begutachten, recht herzlich bedanken. Weiters gilt mein besonderer Dank der e\&i-Redaktion für die ausgezeichnete Zusammenarbeit!

Seit ich im Jahr 2006 zum ersten Mal ein e\&i-Heft zum Thema elektromagnetische Verträglichkeit (EMV) organisieren durfte, ist die EMV in der Industrie mehr denn je zu einer Schlüsseltechnologie für die erfolgreiche Entwicklung elektrischer und elektronischer Baugruppen und Geräte, Systeme und Anlagen sowie integrierter Schaltungen geworden.

Der Themenschwerpunkt der damaligen Ausgabe 1-2.2006 lautete "10 Jahre EMV gesetzlich verpflichtend", da die erste EMVRichtlinie mit 1.1.1996 für alle elektrischen und elektronischen Geräte, die in der Europäischen Union auf den Markt gebracht werden, gesetzlich verpflichtend wurde. Aktuell ist die 3. Ausgabe der EMV-
Richtlinie, die "Richtlinie 2014/30/EU des Europäischen Parlaments und des Rates vom 26. Februar 2014 zur Harmonisierung der Rechtsvorschriften der Mitgliedstaaten über die elektromagnetische Verträglichkeit" in Kraft. Sie wurde mit der EMVV 2015 (elektromagnetische Verträglichkeitsverordnung) in österreichisches Recht umgesetzt, wobei die allgemeinen Teile im ETG 1992 idF Novelle 2017 umgesetzt sind.

Ich bin davon überzeugt, dass das Thema EMV in Zukunft wichtig bleiben bzw. noch mehr an Bedeutung erlangen wird, wenn man nur beispielsweise an die zunehmende Komplexität elektrischer/elektronischer Systeme und an die wachsende Zahl an Funkdiensten denkt.

Noch kurz ein paar Worte zur Entwicklung der EMV-Fachtagung: Sie wird seit 2003 abwechselnd an der TU Graz und am TechCampus Seibersdorf abgehalten. Die Veranstaltung hat sich in den letzten 18 Jahren zu einem „Familientreffen" der österreichischen EMV-Community entwickelt und ist inzwischen über die Grenzen Österreichs hinaus bekannt. Sie dient dem Erfahrungsaustausch in den Bereichen Elektronikentwicklung, Messgeräte- und Entstörbauteileherstellung, von EMV-Fachleuten sowie Manager/innen und Entscheidungsträger/innen aus der Wirtschaft und von Universitäten und Behörden.

Das zweitägige Programm ist auch 2020 wieder thematisch breit gefächert und deckt viele aktuelle Aspekte aus den Bereichen Forschung, Entwicklung, Herstellung und Anwendung ab. Das Tagungsprogramm umfasst 13 Vorträge, zwei Praxisworkshops und eine Poster-Session. Parallel zur Tagung wird wieder eine große Fachausstellung organisiert, für die nationale und internationale Firmen gewonnen werden konnten. Darüber hinaus wird eine Führung durch das akkreditierte EMV-Prüfzentrum angeboten.

Ich wünsche Ihnen, sehr geehrte Leserinnen und Leser, viel Vergnügen bei der Lektüre der Beiträge und es würde mich freuen, Sie bei der EMV-Fachtagung begrüßen zu dürfen!

Das Programm der EMV-Fachtagung und die Anmeldeplattform sind auf der Fachtagungs-Webpage zu finden: www.emvfachtagung.at.

Hinweis des Verlags Der Verlag bleibt in Hinblick auf geografische Zuordnungen und Gebietsbezeichnungen in veröffentlichten Karten und Institutsadressen neutral.
Lamedschwandner, Kurt, Leiter des Fachbereichs EMV der Seibersdorf Labor GmbH 2444 Seibersdorf, Österreich (E-Mail: kurt.lamedschwandner@seibersdorf-laboratories.at) 